\title{
Prevalence of cartilage lesions in foot joints: a test of the concept of limited and progressive lesions
}

\author{
H R YOUNGMAN, ${ }^{1}$ F COOPER, ${ }^{1}$ AND PAUL BYERS ${ }^{2}$ \\ From the ${ }^{1}$ London Foot Hospital, London; and the ${ }^{2}$ Institute of Orthopaedics (University of London), Royal \\ National Orthopaedic Hospital, Stanmore, Middlesex
}

SUMMARY Lesions in the articular cartilage of the joints of the first ray of feet amputated from 54 patients in the age range 10-79 years have been categorised by site, size, and stage of development. The large number of small lesions in early stages of development, appearing from an early age, and the few lesions with extensive bone exposure, occurring only in later years, lend corroboration to the concept of limited and progressive lesions, as established for the hip joint.

Key words: articular cartilage, osteoarthritis.

In 1970 Byers et al applied criteria of location and macroscopic appearance, including fine detail $x$ ray, to categorise lesions in the articular cartilage of 365 right hip joints. ${ }^{1}$ Although they produced an elaborate classification of lesions, there was no fundamental difference in anatomical location from that already described by others. ${ }^{2-4}$ They made the distinction, however, between progressive lesions (osteoarthritis) and those of limited progression. 'Progressive' implies a continuing loss of cartilage at a given site leading to bone exposure and joint deformity. 'Limited progress' connotes lesions that cease to progress before bone is exposed and do not lead to deformity. The basis of the distinction was by site, macroscopic appearance, and the ultimate fate of the lesions, and the knowledge of the clinical prevalence of osteoarthritis (OA). Perifoveal alterations in the cartilage and those at the outer margin were far more prevalent than the clinical incidence of $\mathrm{OA}$ and never progressed to bone exposure and joint deformity. Lesions on the inferior surface were also common and progressed occasionally. Anterosuperior lesions were progressive, and their prevalence was similar to that of clinical OA. The 365 right hip joints in that study were a sufficiently large number to establish statistically that the two classes of lesion were independent. ${ }^{7}$ It was possible also to demonstrate one histological feature, a very fine fibrillation, that was peculiar to early progressive lesions. $^{5}$

Accepted for publication 13 March 1987.

Correspondence to Dr Paul Byers, Institute of Orthopaedics (University of London), Royal National Orthopaedic Hospital, Stanmore, Middlesex HA7 4LP.
Further corroboration of the validity of distinguishing between the two categories was found in studies of femoral heads from populations that are recognised not to experience OA of the hip: patients with fractured femoral necks and Asians. ${ }^{6}$ Lesions were found in these which were identical to the limited category of the postmortem study, but none corresponded to the progressive category.

The concept of limited and progressive lesions has never been tested in any other joint. Feet from amputations offer an opportunity to examine a large number of joints, in only a few of which is $O A$ a clinical problem. The prevalence of $\mathrm{OA}$ in foot joints is not recorded, in contrast with data for the hip joint. Nevertheless, it seems to be generally accepted that OA of the first metatarsophalangeal (MTP) joint in Caucasians is not uncommon, particularly in the presence of hallux valgus. Other joints rarely come to attention. It is also accepted that in Middle East populations OA of the feet, if not non-existent, is very rare. The expectation therefore was that in only a few first MTP joints in older Caucasians would there be lesions which had progressed to bone exposure over large areas (OA); by contrast a larger number of small peripheral lesions would be expected in all joints, beginning at an early age. On the basis of experience with Asian hip joints it was expected that in the feet of the Middle East patients no OA lesions would be found, but that limited lesions similar to those in Western feet would be present.

\section{Materials and methods}

Feet from amputated legs have been collected over a 
Table 1 Age distribution of the patients and (the number of Asians)*

\begin{tabular}{lc}
\hline Age & Number \\
\hline $0-9$ & 0 \\
$10-19$ & $14(4)$ \\
$20-29$ & $12(4)$ \\
$30-39$ & $6(1)$ \\
$40-49$ & 5 \\
$50-59$ & $10(1)$ \\
$60-69$ & $5(1)$ \\
$70-79$ & 1
\end{tabular}

*The age of one patient was not known.

number of years. Fifty four were used, mainly from amputations carried out in the treatment of neoplasia. In none had the feet been directly involved. The age distribution of the patients is given in Table 1 . The specimens were examined by two observers working together. At the outset a few specimens were examined on several occasions to familiarise the observers with the kind of alterations to be found and to check on their consistency, but a formal observer error anlaysis was not carried out. The location, character, and size of alterations in the cartilage were recorded. Particular attention was paid to identifying bone exposure. The criteria for size (the proportion of the surface affected by the aggregate of lesions) and stage of development (which is an expression of the depth) of lesions are listed in Tables 3 and 4. Nine articular surfaces were studied in each foot: first cuneiform metatarsal; first MTP; second MTP; first metatarsal sesamoid (only the plantar surface of the metatarsal); first interphalangeal (IP) joint.

\section{Results}

The age distribution of the patients was maximal ats 10-19 years (14 cases), with a second, lower peak af 20-29 years ( 12 cases), and a third at $50-59$ years (10E cases). The age distribution of all the patients and ote those from the Middle East is given in Table 1. Ther population sample was not adequate to establish accurately the age prevalence of lesions in the articular cartilages examined, but sufficient to show $\overrightarrow{0}$ that alterations appear at an early age and increase with time. About $60 \%$ of the joint surfaces has acquired lesions by the third decade; more advancedo stages were seen in the later decades. Nearly all of these lesions were peripheral, affecting $25-50 \%$ of the surface. In only three joints had lesions giveri rise to extensive central bone exposure of a degree indicative of progressive cartilage destruction.

The findings are shown in Tables 2 to 5 , which record the number, distribution, and stage of lesions.

Every class of articular surface is affected to some degree: least affected was the distal IP joint, in onl ${ }_{00}^{\circ}$ five of which were lesions detected. Most affected ${ }^{N}$ was the plantar surface of the first metatarsal joint treated separately because of its articulation witls the sesamoid, only six of which were unaltered. Thi was followed by the base of the first phalanx, 44 of which were affected.

Most alterations were small. More than half of the surface was affected in only 19 ; which reduced to 19 when the plantar surface of the first metatarsal was excluded.

Equally, advanced lesions were uncommon. Stage 4 was recorded five times; and stage 5 (bons exposure) three times - both surfaces of the firsi MTP joint in a woman of 54 years; and the proximap.

Table 2 Distribution of lesions on the various joint surfaces

\begin{tabular}{|c|c|c|c|c|c|c|c|c|c|}
\hline \multirow[t]{2}{*}{ Site } & \multicolumn{2}{|c|}{$C M^{*}$} & \multicolumn{2}{|c|}{ lst $M T P^{*}$} & \multicolumn{2}{|c|}{ 2nd $M T P$} & \multicolumn{2}{|c|}{$I P^{*}$} & \multirow[t]{2}{*}{$M T / S^{*}$} \\
\hline & $p$ & $d$ & $p$ & $d$ & $p$ & $d$ & $p$ & $d$ & \\
\hline No lesion & 28 & 23 & 13 & 11 & 37 & 52 & 42 & 50 & 6 \\
\hline Central & 8 & 8 & 15 & 35 & 1 & 0 & 1 & 2 & 13 \\
\hline \multicolumn{10}{|l|}{ Peripheral } \\
\hline Plantar & 5 & 6 & 13 & 15 & 5 & 2 & 3 & 2 & 38 \\
\hline Dorsal & 4 & 4 & 14 & 8 & 3 & 1 & 4 & 3 & 38 \\
\hline Medial & 17 & 20 & 19 & 8 & 9 & 0 & 2 & 2 & 2 \\
\hline Lateral & 11 & 11 & 9 & 6 & 1 & 0 & 7 & 1 & 20 \\
\hline Others & 0 & 0 & 19 & 0 & 1 & 0 & 7 & 1 & 20 \\
\hline
\end{tabular}

The two columns for each joint represent the proximal and distal surfaces, except for the metatarsal sesamoid, where only the metatarsal surface was scored. Since there were several lesions in many joints the totals are greater than the surfaces. In some joints the location of lesions was difficult to define precisely according to the method employed; they were put in category 6 (others).

${ }^{*} \mathrm{CM}=$ cuneometatarsal; $\mathrm{MTP}=$ metatarsophalangeal; $\mathbf{I P}=$ interphalangeal; $\mathrm{MT} / \mathrm{S}=$ metatarsal sesamoid; $\mathrm{d}=\mathrm{distal} ; \mathrm{p}=\mathrm{proximal}$. 
Table 3 Distribution by size of the largest lesion in each joint surface*

\begin{tabular}{|c|c|c|c|c|c|c|c|c|c|}
\hline \multirow[t]{2}{*}{ Size } & \multicolumn{2}{|c|}{$C M^{*}$} & \multicolumn{2}{|c|}{ lst $M T P^{*}$} & \multicolumn{2}{|c|}{ 2nd $M T P$} & \multicolumn{2}{|l|}{$I P^{*}$} & \multirow[t]{2}{*}{$M T / S$} \\
\hline & $p^{*}$ & $d^{*}$ & $p$ & $d$ & $p$ & $d$ & $p$ & $d$ & \\
\hline $\begin{array}{l}\text { Speck } \\
\text { Percentage of the } \\
\text { total surface: }\end{array}$ & 7 & 13 & 7 & 8 & 6 & 2 & 5 & 0 & 5 \\
\hline 10 & 14 & 13 & 20 & 15 & 6 & 2 & 6 & 2 & 18 \\
\hline 25 & 5 & 5 & 10 & 11 & 2 & 0 & 3 & 2 & 17 \\
\hline 50 & 1 & 1 & 1 & 2 & 0 & 0 & 0 & 0 & 4 \\
\hline 75 & 0 & 0 & 2 & 1 & 0 & 0 & 0 & 0 & 5 \\
\hline 100 & 0 & 0 & 1 & 1 & 0 & 0 & 0 & 0 & 0 \\
\hline
\end{tabular}

${ }^{*}$ For abbreviations see Table 2.

phalangeal surface of a 53 year old man. The whole of these three surfaces were denuded; such lesions would qualify as progressive, i.e., OA.

Most alterations were in the periphery of the joint. Centrally located lesions were found mainly in the first MTP joint, and here the base of the phalanx was affected twice as frequently as the metatarsal head. This lesion, not ordinarily associated with bone exposure, is regularly observed in material removed in the treatment of hallux valgus.

The sesamoid surfaces were not easy to analyse, nor was this necessary for the purpose of this study. It was noted that 31 of the sesamoids were free of blemish, and that most of the remainder had only minimal changes. One joint was fused, and in another there was bone exposure. The remainder had minor alterations.

A more detailed presentation of the occurrence and distribution of lesions is given in Table 5. Here the maximum stage achieved by whatever lesions are present is recorded according to age by decades. Since a single specimen should have nine surfaces the number of surfaces in any decade is $9 \times$ No of patients. Since the age of one patient was not known, however, and the changes in nine joints were not completely recorded not all the figures in every row and each column add up to the theoretical total. There is an additional clerical error in that two extra joint surfaces appear in the decade $40-49$ years and four in the decade 60-69 years; these have defied explanation and we have avoided "creative accounting' to eliminate them. The number of Middle East joint surfaces in a category have been indicated in parentheses. This table shows the age related nature of the lesions. Lesions were detected in the second decade regardless of race. Overall the proportion of unaffected joint surfaces was similar at roughly $55 \%$. That $45 \%$ of these joint surfaces should be affected by OA is more than can reasonably be accepted. The natural history of these lesions, as deduced from this material, conforms to the concept of limited progression.

\section{Discussion}

This study was not an attempt to describe the detailed pathology of the articular cartilage lesions. The concept of lesions of limited progression-this term, originally employed by Byers et al $1970,{ }^{1}$ has by usage become equated with 'non-progressive', which is not entirely accurate-implies that they evolve, and hence can be graded in stages of

Table 4 Distribution by stage of the most developed lesion in each surface

\begin{tabular}{|c|c|c|c|c|c|c|c|c|c|}
\hline \multirow[t]{2}{*}{ Stage } & \multicolumn{2}{|c|}{$C M$} & \multicolumn{2}{|c|}{ lst $M T P$} & \multicolumn{2}{|c|}{ 2nd $M T P$} & \multicolumn{2}{|l|}{$I P$} & \multirow[t]{2}{*}{$M T / S$} \\
\hline & $p$ & $d$ & $p$ & $d$ & $p$ & $d$ & $p$ & $d$ & \\
\hline $\begin{array}{l}\text { Discoloration } \\
\text { Tissue loss: }\end{array}$ & 5 & 6 & 12 & 19 & 10 & 1 & 10 & 10 & 5 \\
\hline Superficial & 18 & 22 & 17 & 13 & 2 & 2 & 11 & 1 & 20 \\
\hline Deeper & 3 & 3 & 8 & 3 & 2 & 0 & 3 & 3 & 21 \\
\hline Very deep & 1 & 0 & 2 & 0 & 0 & 0 & 0 & 0 & 2 \\
\hline Bone exposed & 0 & 0 & 2 & 1 & 0 & 0 & 0 & 0 & 0 \\
\hline
\end{tabular}

For abbreviations see Table 2. 
development, but that the development is arrested before bone is exposed, and joint function is not impaired to the point where treatment is undertaken. Hence, there is also the implication that they do not occur in critical areas, which by inference means they are found at the margins, leaving an intact central region for load bearing. In the hip substantiation of these inferences lay not only in the observation of the lesions but also in their prevalence, which exceeded that of individuals undergoing treatment for a clinical diagnosis of $\mathrm{OA}$. The location and limited development of most foot joint lesions compares positively with experience in the hip. The use of prevalence data of OA in the feet is difficult since there are no accurate figures, but clinically only a few classes of foot joint give rise to complaints that are diagnosed as OA.

Although this study has not been extended to a detailed pathological description of the lesions, the observations are sufficient to establish that there are lesions of limited progression that do not qualify as osteoarthritic, and the hypothesis of limited and progressive lesions of articular cartilage has been given a degree of corroboration.

Two small points need comment. In the hip joint study a small number of early $\mathrm{OA}$ lesions were observed. There has been no such finding in the current study. This is accounted for by chance, owing to the relatively small number of specimens.

The central lesions in the proximal surface of the first phalanx, which have been studied in material from the surgical treatment of hallux valgus, are microscopically similar to some of the changes observed in the apex of the acetabulum, and are equally mysterious. Byers $e t$ al in 1970 attributed the acetabular lesion to a residual effect of the triradiate cartilage,${ }^{1}$ though there is an argument that it is related to absence of loading due to joint incongruency. The former argument does not apply to the phalanx, but the second might.

We conclude that the natural history of these lesions, as deduced from this material, corroborates the concept of limited progression versus progressive lesions. It suggests, as in the hip joint, that the two are independent, although here, in contrast with that study, ${ }^{7}$ no statistical analysis has been undertaken.

\section{References}

1 Byers P D. Contepomi C A. Farkas T A. A postmortem study of the hip joint. Ann Rheum Dis 1970; 29: 15-31.

2 Harrison M H M. Schaiowicz F. Trueta J. Osteoarthritis of the hip: a study of the nature and evolution of the disease. $J$ Bone Joint Surg /Brl 1953; 35B: 598-626.

3 Heine J. Uber die Osteoarthritis Deformans. Virchows Arch [A] 1926; 260: 621 .

4 Goodfellow J, Bullough P G. Studies on age changes in human hip joint. J Bone Joint Surg /Br/ 1968; 50B: 222.

5 Byers P D, Contepomi C A. Farkas T A. A postmortem study of the hip joint. II. The histological basis for limited and progressive lesions. Ann Rheum Dis 1976; 35: 114-21.

6 Byers P D, Hoagland F T. Purewal G R, et al. Articular cartilage changes in Caucasian and Asian hip joints. Ann Rheum Dis 1974: 33: 157-61.

7 Byers P D. Contepomi C A. Farkas T A. A postmortem study of the hip joint. III. Correlations between observations. Ann Rheum Dis 1976; 35: 122-6. 